\title{
Complexity and Online Algorithms for Minimum Skyline Coloring of Intervals ${ }^{\text {th }}$
}

\author{
Thomas Erlebach ${ }^{1}$ \\ Department of Informatics, University of Leicester, UK \\ Fu-Hong Liu \\ Department of Computer Science, National Tsing Hua University, Taiwan \\ Hsiang-Hsuan $\mathrm{Liu}^{2}$ \\ Institute of Computer Science, University of Wroctaw, Poland \\ Mordechai Shalom \\ TelHai College, Upper Galilee, 12210, Israel \\ Prudence W.H. Wong ${ }^{3}$ \\ Department of Computer Science, University of Liverpool, UK \\ Shmuel Zaks \\ Department of Computer Science, Technion, Haifa, Israel \\ School of Engineering, Ruppin Academic Center, Israel
}

\footnotetext{
${ }^{1}$ A preliminary version of this paper has been presented at the 11th International Conference on Combinatorial Optimization and Applications (COCOA 2017).

Email addresses: t.erlebach@leicester.ac.uk (Thomas Erlebach), fhliu@cs.nthu.edu.tw (Fu-Hong Liu), alison.hhliu@cs.uni.wroc.pl (Hsiang-Hsuan Liu), cmshalom@telhai.ac.il (Mordechai Shalom), pwong@liverpool.ac.uk (Prudence W.H. Wong), zaks@cs.technion.ac.il (Shmuel Zaks)

${ }^{1}$ Supported by a study leave granted by University of Leicester.

${ }^{2}$ Partially supported by Polish National Science Centre grant 2016/22/E/ST6/00499 and partially supported by a Dual PhD studentship when the author was with University of Liverpool and National Tsing Hua University.

${ }^{3}$ Partially supported by the initiative Networks Sciences \& Technologies (NeST) by School of EEECS, University of Liverpool.
} 


\begin{abstract}
Motivated by applications in optical networks and job scheduling, we consider the interval coloring problem in a setting where an increasing cost is associated with using a higher color index. The cost of a coloring at any point of the line is the cost of the maximum color index used at that point, and the cost of the overall coloring is the integral of the cost over all points on the line. A coloring of minimum cost is called a minimum skyline coloring. We prove that the problem of computing a minimum skyline coloring is NP-hard and initiate the study of the online setting, where intervals arrive one by one. We give an asymptocially optimal online algorithm for the case of linear color costs and present further results for some variations and generalizations of the problem. Furthermore, we consider the variant of the minimum skyline coloring problem where the intervals are already partitioned into color classes and we only need permute the colors so as to minimize the cost of the coloring. We show that this problem variant is NP-hard and present a 2-approximation algorithm for it.
\end{abstract}

Keywords: Skyline coloring of intervals, online algorithm, NP-hardness, approximation algorithm.

\title{
1. Introduction
}

Graph coloring has been studied extensively in the literature [16]. In the basic problem, given a graph we have to color its vertices such that no two adjacent vertices are assigned the same color. The classical version of the problem concerns the number of colors used. Many different variants of the problem have been studied, e.g., coloring edges instead of vertices, focusing on different graph classes, and concerning different objective functions [7, 11, 12, 15, 16, 19, 20, 23.

In this paper, we focus on coloring of intervals [14] in which the input is a set of intervals on a line and two overlapping intervals cannot be assigned the same color. This corresponds to a coloring of an interval graph in the classical sense, however our cost measure is different, as follows. We are interested in the setting where there is an increasing cost associated with using a higher color index. Given a set of intervals (represented on a line) and a coloring, the cost of the coloring at any point is the cost of the maximum color index used at that point and the overall cost of the coloring is the integral of the cost over all points on the line. Intuitively, the maximum color index used 
at each point forms a "skyline" and so the objective is to obtain a minimum skyline coloring. A formal definition of skyline will be given in Section 2 .

The problem arises in various applications. In communication networks like optical networks in the line topology, a network needs to be equipped with optical amplifier devices for transmitting data through the optical fiber. The devices are increasingly more complicated when we need a higher wavelength (cf. color), and hence require a higher cost to operate; and each type of amplifier device is capable of amplifying all the wavelengths up to a certain maximum. Therefore, the cost of operation depends on the maximum wavelength which is reflected in the cost of the maximum color index defined in our problem. See [2] for a more detailed discussion.

Another application is from job scheduling, where each job has a required execution interval and has to be assigned to a machine. The machines are in an ordered list and one must at any time hire a set of machines that is a prefix of that ordered list. This means that if the machine of the largest index that one currently uses is machine $k$, one must pay the rental cost for the first $k$ machines.

Related work. Problems concerning coloring of intervals were first studied with the objective of minimizing the number of colors used [14]. Generalizations considered include minimizing the sum of the colors assigned to the vertices [11, 12, 15, 19]; incorporating a bandwidth requirement for each interval and allowing overlapping intervals to be assigned the same color as long as their total bandwidth requirement does not exceed the capacity [1, 3]. The work most relevant to this paper includes generalized coloring problems studied in [2, 17, 23] and the busy time scheduling problems [5, 6, 8, 13, 18, 22,

In [2], the following more general problem of coloring intervals is defined. The set of colors is partitioned into color classes and each color class $C_{i}$ has a cost of $i$. At any point on the line, if a color in $C_{i}$ is the largest color assigned to some interval containing the point, then the cost at this point is $i$. The authors prove that this problem is NP-hard via a reduction from Numerical Three Dimensional Matching. This reduction requires that some color class contains more than one color. A 2-approximation algorithm is also proposed in the paper. In the busy time scheduling problem [5, 6, 8, 13, 18, 22], a machine (cf. color) can be shared by a certain number of jobs (cf. intervals) and the usage of a machine costs the same no matter how many jobs are sharing the machine. The busy time problem can also be presented as other equivalent problems, e.g., in the context of optical line network wavelength assignment [17, 23] and dynamic bin packing with minimum server usage 
time [21].

Our contribution. The problem we study in this paper is a special case of the problem in [2] in which each color class consists of one color. Yet we prove a stronger NP-hardness result revealing that the problem remains NP-hard (Section 3). The proof is via a reduction from the ARCCOLORING problem [10] and works for any strictly increasing color cost function. We then initiate the study of the online setting for the problem (Section 4). For the case where the cost of a color is proportional to its index, we present an $O\left(\log \frac{\ell_{\max }}{\ell_{\min }}\right)$-competitive algorithm, where $\ell_{\max }$ and $\ell_{\min }$ are the maximum and minimum length of the intervals. The algorithm assumes the knowledge of $\frac{\ell_{\max }}{\ell_{\min }}$ in advance. We also show a lower bound of $\frac{1}{2} \log \frac{\ell_{\max }}{\ell_{\min }}$ on the competitive ratio for any deterministic online algorithm even when the algorithm knows $\frac{\ell_{\max }}{\ell_{\min }}$ in advance. This implies that our online algorithm is asymptotically optimal. In addition, we extend our results to the case when each color has a positive capacity $\kappa$ and can be assigned to a set of intervals with load at most $\kappa$ (Section 5.1) showing that the online algorithm applies with only a constant factor increase in the competitive ratio. On the other hand, if the cost function is an arbitrary increasing function instead of linear in the class index, then any deterministic online algorithm can perform very badly (Section 5.2). We also note that our online algorithm applies when the underlying graph is a circular graph instead of a line (Section 5.3).

The coloring problem essentially consists of two components: partitioning the intervals into disjoint subsets such that in each subset no two intervals overlap; and assigning a color to each subset. We consider a variant of this problem in which the subsets are given and the only decision is to assign a different color to each subset (Section 6), i.e., find a permutation of the subsets to map to color $1,2, \cdots$. At first glance this permutation problem may sound easier. Nevertheless, we show that the permutation problem is NP-hard (Section 6.1) by presenting a reduction from the optimal linear arrangement problem [9]. We also design a 2-approximation algorithm for the permutation problem (Section 6.2).

\section{Definitions and preliminaries}

Problem definition. We are given a set of $n$ intervals $\mathcal{I}=\left\{I_{1}, I_{2}, \cdots, I_{n}\right\}$. Each $I_{j}$ is a half open interval $\left[s_{j}, e_{j}\right)$, where $s_{j}$ and $e_{j}$ denote real numbers that are the start and end point of the interval $I_{j}$, respectively. Two intervals 
$I_{i}$ and $I_{j}$ are overlapping if $I_{i} \cap I_{j} \neq \emptyset$. Interval $I_{j}$ contains point $t$ if $t \in I_{j}$, i.e., $s_{j} \leq t<e_{j}$. We denote by $\mathcal{I}_{t}$ the set of intervals of $\mathcal{I}$ that contain point $t$, i.e., $\mathcal{I}_{t}=\left\{I_{j} \in \mathcal{I} \mid I_{j} \ni t\right\}$, and by $\operatorname{load}_{\mathcal{I}}(t)$ the number $\left|\mathcal{I}_{t}\right|$ of these intervals which is termed the load induced by $\mathcal{I}$ at point $t$. When there is no ambiguity, we omit the subscript $\mathcal{I}$ and simply write $\operatorname{load}(t)$. The length of $I_{j}$, denoted by $\ell\left(I_{j}\right)$, is defined as $e_{j}-s_{j}$. The maximum and minimum lengths over all intervals in $\mathcal{I}$ are denoted by $\ell_{\max }$ and $\ell_{\min }$, respectively. The length $\ell(\mathcal{S})$ of a set $\mathcal{S}$ of intervals is the sum of the lengths of all intervals in $\mathcal{S}$, i.e., $\ell(\mathcal{S})=\sum_{I \in \mathcal{S}} \ell(I)$.

We are also given an infinite set of colors $\Lambda=\{1,2,3, \cdots\}$, and every color $i$ has an associated cost $\lambda(i) \geq 1$, where $\lambda$ is a non-decreasing function of $i$. A coloring $\omega: \mathcal{I} \rightarrow \Lambda$ is valid if for any pair of distinct overlapping intervals $I_{i}$ and $I_{j}$, we have $\omega\left(I_{i}\right) \neq \omega\left(I_{j}\right)$. We refer to the coloring of the intervals in $\mathcal{I}$ as $\omega(\mathcal{I})$. For any subset $\mathcal{I}^{\prime} \subseteq \mathcal{I}$, we denote by $\omega\left(\mathcal{I}^{\prime}\right)$ the coloring obtained by restricting $\omega$ to the intervals $\mathcal{I}^{\prime}$. The instantaneous cost of $\omega$ at point $t$, denoted by $\operatorname{cost}(\omega, t)$, is the maximum cost of the colors of all intervals containing $t$, i.e., $\operatorname{cost}(\omega, t)=\max _{I \in \mathcal{I}_{t}} \lambda(\omega(I))$ if $\mathcal{I}_{t} \neq \emptyset$ and zero otherwise. Note that $\operatorname{cost}(\omega, t)=0$ when $\operatorname{load}(t)=0$. Since $\lambda$ is non-decreasing, we have $\operatorname{cost}(\omega, t)=\lambda\left(\max _{I \in \mathcal{I}_{t}} \omega(I)\right)$. We term this color (i.e., $\left.\max _{I \in \mathcal{I}_{t}} \omega(I)\right)$, as the skyline of $\omega$ at $t$, and the unique interval of $\mathcal{I}_{t}$ colored with this color, as the contributing interval of $\omega$ at $t$. We denote the set of all contributing intervals by $\mathcal{I}_{\mathrm{s}}$, i.e., an interval $I$ is in $\mathcal{I}_{\mathrm{s}}$ if there exists $t \in I$ such that $\operatorname{cost}(\omega, t)=\lambda(\omega(I))$, or equivalently, $I=\arg \max _{I \in \mathcal{I}_{t}} \omega(I)$.

The total cost of $\omega$, denoted as $\operatorname{cost}(\omega)$, is the integral of all the instantaneous costs, i.e., $\operatorname{cost}(\omega)=\int_{-\infty}^{\infty} \operatorname{cost}(\omega, t) \mathrm{d} t$. From our definitions it follows that $\operatorname{cost}(\omega(\mathcal{I}))=\operatorname{cost}\left(\omega\left(\mathcal{I}_{\mathrm{s}}\right)\right)$. Moreover, when $\omega$ is a valid coloring we have $\max _{I \in \mathcal{I}_{t}} \omega(I) \geq \operatorname{load}(t)$, since the intervals of $\mathcal{I}_{t}$ are colored with distinct colors. Therefore, $\operatorname{cost}(\omega, t) \geq \lambda(\operatorname{load}(t))$, and consequently, $\operatorname{cost}(\omega) \geq \int_{-\infty}^{\infty} \lambda(\operatorname{load}(t)) \mathrm{d} t$. A valid coloring for which the last inequality is tight is clearly optimal. We term such colorings as load-optimal. From the definitions it follows:

Observation 1. Let $\lambda$ be any strictly increasing color cost function. A valid coloring $\omega$ of an input set of intervals $\mathcal{I}$ is load-optimal if and only if for every point $t$, the set of colors used by $\omega$ for intervals in $\mathcal{I}_{t}$ is $\{1, \ldots, \operatorname{load}(t)\}$.

In this work, unless otherwise specified, we assume $\lambda(i)=i$. Whenever this is the case we have $\int_{-\infty}^{\infty} \lambda(\operatorname{load}(t)) \mathrm{d} t=\int_{-\infty}^{\infty} \operatorname{load}(t) \mathrm{d} t=\ell(\mathcal{I})$. The last 


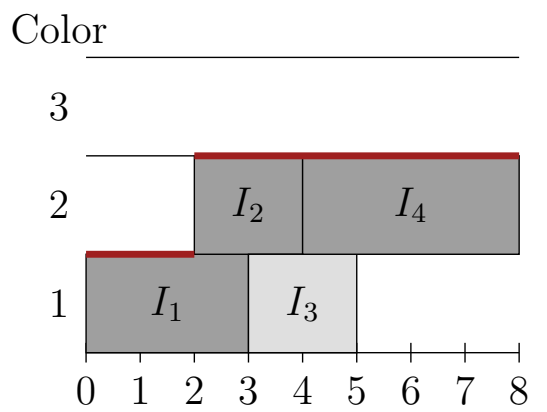

(a) Assigning the lowest available color. The cost is $2 \times 1+6 \times 2=14$.

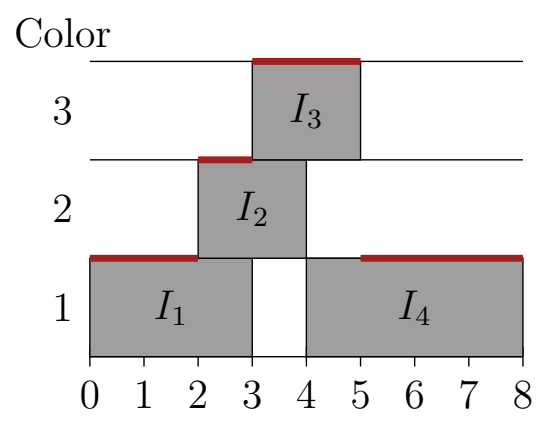

(b) Optimal coloring. The cost is $5 \times$ $1+1 \times 2+2 \times 3=13$.

Figure 1: Two different colorings of four intervals. An optimal solution does not necessarily minimize the number of colors used. The darker intervals contribute to the cost of the coloring but the lighter interval does not. The bolded line indicates the skyline.

equality is due to the fact that every infinitesimal subinterval of an interval in $\mathcal{I}$ contributes the same value (namely, its length) to both sides. This implies:

Observation 2. For every valid coloring $\omega$ of a set $\mathcal{I}$ of intervals, we have $\operatorname{cost}(\omega) \geq \ell(\mathcal{I})$ when $\lambda(i)=i$ for all $i$.

The objective of the SKYLINE problem is to find a valid coloring $\omega$ such that $\operatorname{cost}(\omega)$ is minimized. Without loss of generality, we can assume that the union $\cup \mathcal{I}$ of the intervals in $\mathcal{I}$ is an interval that we term the horizon. Otherwise, the coloring of each maximal interval of $\cup \mathcal{I}$ is independent of the others. Figure 1 illustrates various notions used in the problem definition.

Approximation and online algorithms. An algorithm for the off-line version of the SKYLINE problem is a $\rho$-approximation algorithm if it runs in polynomial time and outputs a valid coloring whose cost is no more than $\rho$ times the cost of an optimal coloring. We also consider the online setting where intervals arrive one at a time in an arbitrary order. An online algorithm has to decide on the color of an interval upon its arrival, and this decision cannot be modified later. Such an algorithm is c-competitive if the cost of the solution of the algorithm is no more than $c$ times that of an optimal (offline) solution, for every input [4. We also denote by $\mathcal{A}$ the coloring returned by an algorithm $\mathcal{A}$, and the cost of this solution by $\operatorname{cost}(\mathcal{A})$. We denote by $\mathcal{O}$ an optimal solution. 


\section{NP-hardness of SKYLINE}

Theorem 3. It is NP-complete to decide whether a given instance of SKYLINE has a load-optimal coloring.

Proof. It is easy to see that the problem is in NP because one can check in polynomial time whether a given coloring is valid and load-optimal. The NP-hardness is proved by a reduction from ARCCOLORING. An instance of ArCColoring is given by a family $\mathcal{F}=\left\{A_{1}, \ldots, A_{n}\right\}$ of circular arcs and a positive integer $K$. Each arc $A_{i} \in \mathcal{F}$ is given by a pair $\left(a_{i}, b_{i}\right)$ with $a_{i} \neq b_{i}$ and $a_{i}, b_{i} \in\{1, \ldots, m\}$ for some $m \leq 2 n$. Intuitively, the set $\{1, \ldots, m\}$ represents points that are located around a circle. The span of arc $A_{i}$ is the set $\left\{a_{i}, a_{i}+1, \ldots, b_{i}-1\right\}$ if $a_{i}<b_{i}$ and $\left\{a_{i}, a_{i}+1, \ldots, m\right\} \cup\left\{1, \ldots, b_{i}-1\right\}$ if $b_{i}<a_{i}$. We say that two arcs intersect if their spans have a non-empty intersection. It is NP-hard to decide whether the arcs in $\mathcal{F}$ can be colored with at most $K$ colors in such a way that arcs with the same color do not intersect [10]. Let an instance $(\mathcal{F}, K)$ of ARCCOLORING be given. We say that a point $p \in\{1, \ldots, m\}$ is contained in an arc if it is contained in the span of the arc. Without loss of generality, we can assume that every point is contained in exactly $K$ arcs: If a point is contained in more than $K$ arcs, the instance is trivially a no-instance. If a point $p$ is contained in fewer than $K$ arcs, we can add arcs of the form $(p, p+1)$ until $p$ is contained in $K$ arcs, without changing the $K$-colorability of the instance.

We construct an instance $\mathcal{I}$ of SKYLINE from $(\mathcal{F}, K)$ as follows. Intuitively, we "cut" the ring at the point 1 to turn the set of arcs into a set of intervals. The intervals resulting from arcs that were cut are then extended (into a "left staircase" and a "right staircase") in such a way that the two intervals resulting from the same arc must receive the same color in any load-optimal coloring. Formally, we create intervals from the arcs in $\mathcal{F}$ as follows: Any arc $A_{i}=\left(a_{i}, b_{i}\right)$ that does not contain the point 1 produces the interval $I_{i}=\left[a_{i}, b_{i}\right)$ if $b_{i}>a_{i}$, or the interval $I_{i}=\left[a_{i}, m+1\right)$ if $b_{i}=1$. Let $A_{j_{1}}, \ldots, A_{j_{K}}$ be the $K$ arcs that contain point 1 . For $1 \leq i \leq K$, the arc $A_{j_{i}}=\left(a_{j_{i}}, b_{j_{i}}\right)$ produces two intervals $I_{j_{i}}^{1}$ and $I_{j_{i}}^{2}$ : If $a_{j_{i}}>b_{j_{i}}$, the two intervals are $I_{j_{i}}^{1}=\left[-K+i, b_{i_{j}}\right)$ and $I_{j_{i}}^{2}=\left[a_{i_{j}}, m+2+K-i\right)$. If $a_{j_{i}}=1<b_{j_{i}}$, the two intervals are $I_{j_{i}}^{1}=\left[-K+i, b_{i_{j}}\right)$ and $I_{j_{i}}^{2}=[m+1, m+2+K-i)$. An example of the construction is shown in Figure 2. The arcs in the example are $A_{1}=(5,3)$, $A_{2}=(4,2), A_{3}=(2,4)$ and $A_{4}=(3,5)$, and $K=2 . A_{1}$ and $A_{2}$ contain the point 1 and thus produce two intervals each, while $A_{3}$ and $A_{4}$ produce only 


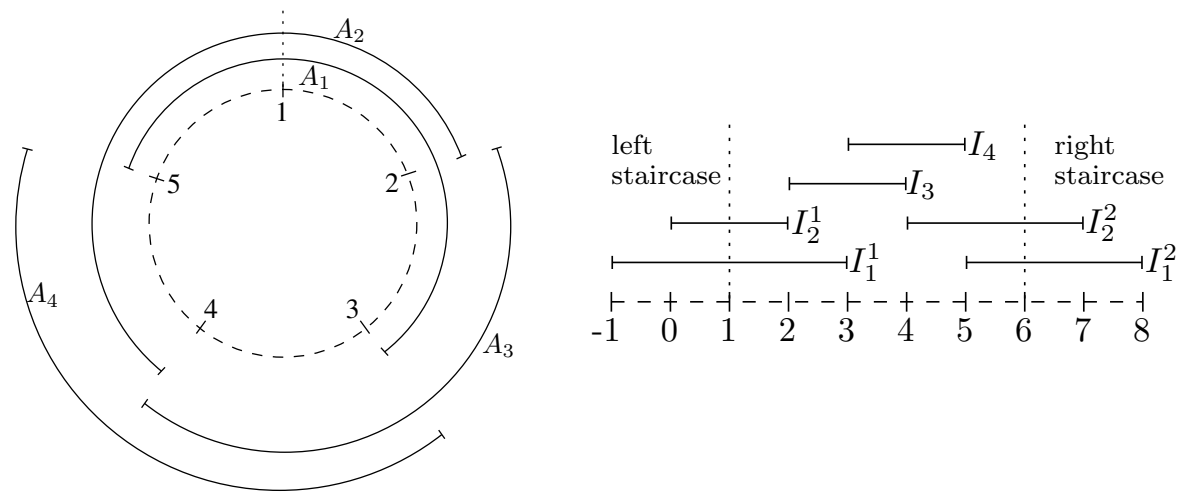

Figure 2: Instance of ARCCOLORING (left), constructed intervals (right)

one interval. In this example, a load-optimal coloring exists: Color $I_{1}^{1}$ and $I_{1}^{2}$ with $1, I_{2}^{1}$ and $I_{2}^{2}$ with $2, I_{3}$ with 2 , and $I_{4}$ with 1 .

We claim that $\mathcal{I}$ has a load-optimal coloring if and only if $(\mathcal{F}, K)$ is a yesinstance of ArcColoring. For the "if" direction, let $\omega: \mathcal{F} \rightarrow\{1, \ldots, K\}$ be a $K$-coloring of $\mathcal{F}$. We can rename the colors so that $\omega\left(A_{j_{i}}\right)=i$ for $1 \leq i \leq K$. Let $\omega^{\prime}: \mathcal{I} \rightarrow\{1, \ldots, K\}$ map each interval in $\mathcal{I}$ to the color assigned by $\omega$ to the arc from which the interval was produced. First, note that $\omega^{\prime}$ is a feasible coloring of $\mathcal{I}$ since any two intervals that intersect are produced from arcs that intersect and hence their colors are different. We claim that $\omega^{\prime}$ is a load-optimal coloring of $\mathcal{I}$. For $t \in[-K+r,-K+r+1)$ for some $r \in\{1,2, \ldots, K\}$, the only intervals containing $t$ are the $r$ intervals $I_{j_{i}}^{1}$ for $1 \leq j \leq r$, and these intervals have colors $1, \ldots, r$. Similarly, for $t \in[m+1+K-r, m+2+K-r)$ for some $r \in\{1,2, \ldots, K\}$, the only intervals containing $t$ are the $r$ intervals $I_{j_{i}}^{2}$ for $1 \leq j \leq r$, and these intervals have colors $1, \ldots, r$. All points $t \in[1, m+1)$ are contained in exactly $K$ intervals that receive colors $1, \ldots, K$. Hence, $\omega^{\prime}$ is indeed load-optimal.

For the "only if" direction, let $\omega^{\prime}$ be a load-optimal coloring of $\mathcal{I}$. The points in $[-K+1,-K+2)$ and $[m+K, m+K+1)$ are contained only in the intervals $I_{j_{1}}^{1}$ and $I_{j_{1}}^{2}$, respectively, and hence these two intervals must both receive color 1 . Similarly, as these intervals form staircase patterns, it follows that $I_{j_{i}}^{1}$ and $I_{j_{i}}^{2}$ must both receive color $i$, for $2 \leq i \leq K$. All other intervals must receive colors in $\{1, \ldots, K\}$ as the load at any point is at most $K$. Define a coloring $\omega: \mathcal{F} \rightarrow\{1, \ldots, K\}$ by assigning to each arc in $\mathcal{F}$ the color of the interval(s) it has produced (for arcs that have produced two intervals, this is still well-defined as both intervals must have the same color, 
as argued above). It follows that $\omega$ is a feasible $K$-coloring of $\mathcal{F}$.

Combining with Observation 1 we have:

Corollary 1. SKYLINE is NP-hard for any strictly increasing color cost function $\lambda$.

\section{Online algorithms for SKYLINE when $\lambda(i)=i$}

In this section, we present online algorithms for the SKYLINE problem for the case where the cost of a color is equal to its index, i.e., $\lambda(i)=i$ for

all $i$. We first focus in Section 4.1 on bounded length intervals and present an $O(1)$-competitive greedy algorithm. In Section 4.2 we adapt the greedy algorithm to the case where the lengths of intervals are arbitrary.

\subsection{Bounded length intervals}

In this section we consider bounded length intervals, i.e., we assume there is a constant $k$ such that for any interval $I$ in the input, we have $\ell(I) \in\left[\ell_{\min }, k \cdot \ell_{\min }\right)$. This section is dedicated to the analysis of the following greedy algorithm.

\subsubsection{The algorithm $\mathcal{G}$ and some basic properties}

When an interval $I_{j} \in \mathcal{I}$ arrives, assign the minimum color that is valid for it, i.e., the minimum color $i$ such that for all $j^{\prime}<j$ and $I_{j^{\prime}} \cap I_{j} \neq \emptyset$, we have $\mathcal{G}\left(I_{j^{\prime}}\right) \neq i$.

Roughly speaking, in the analysis, we select a subset of intervals on the skyline of $\mathcal{G}$ (i.e., from $\mathcal{I}_{\mathrm{s}}$ ), partition the horizon into segments based on this subset, and show that we can "charge" the costs of $\mathcal{G}$ and $\mathcal{O}$ to this subset, thus allowing us to relate the two costs. The partition of the horizon is based on the notion of extended interval. For any interval $I_{j}$, we define its hat interval as $I_{j}^{\mathrm{h}}=\left[s_{j}-k \ell_{\min }, e_{j}+k \ell_{\min }\right)$ and extended hat interval as $I_{j}^{\mathrm{e}}=\left[s_{j}-3 k \ell_{\min }, e_{j}+3 k \ell_{\min }\right)$. Clearly, $\ell\left(I_{j}^{\mathrm{e}}\right)=6 k \ell_{\min }+\ell\left(I_{j}\right) \leq 7 k \ell_{\min }$.

We first observe a property of $\mathcal{G}$. Intuitively, when $\mathcal{G}$ assigns an interval $I$ a certain color $c$, there is a substantial number of intervals overlapping with $I$ in the input. Precisely,

Lemma 1. Consider an interval $I_{j}$ with $\mathcal{G}\left(I_{j}\right)=c$. (i) There are at least $c-1$ intervals that overlap with $I_{j}$ and are contained in $I_{j}^{\mathrm{h}}$; (ii) the total length of these intervals together with $I_{j}$ is at least $c \ell_{\min }$. 
Proof. (i) Since $\mathcal{G}$ assigns the smallest possible color to any interval, $I_{j}$ gets color $c$ only if there are already $c-1$ intervals colored by $1,2, \cdots, c-1$ each of which overlaps with $I_{j}$. Since the length of any interval is bounded by $k \ell_{\text {min }}$, the start point of each such interval is at least $s_{j}-k \ell_{\min }$ and its end point is at most $e_{j}+k \ell_{\min }$, i.e., it is contained in $I_{j}^{\mathrm{h}}$.

(ii) Follows from (i) and the fact the length of any interval is at least $\ell_{\text {min }}$.

\subsubsection{Analysis of $\mathcal{G}$}

Overview. The analysis is based on choosing a subset of intervals $\mathcal{I}_{\mathrm{s}}^{*}$ of $\mathcal{I}_{\mathrm{s}}$. We first give an overview of the role of $\mathcal{I}_{\mathrm{s}}^{*}$ and then show how to construct $\mathcal{I}_{\mathrm{s}}^{*}$. The aim is to obtain the following properties: (i) the hat intervals of any two intervals of $\mathcal{I}_{\mathrm{s}}^{*}$ do not overlap (Lemma 2 (i)), (ii) the union of the extended hat intervals of $\mathcal{I}_{\mathrm{s}}^{*}$ form a contiguous interval that contains the horizon. The first allows us to lower bound $\operatorname{cost}(\mathcal{O})$ by considering these hat intervals, since these hat intervals are disjoint (Lemma 3 (i)). The second property means that we can map each interval to some extended hat interval of $\mathcal{I}_{\mathrm{s}}^{*}$ (Lemma 2 (ii)). As to be shown, the procedure of selecting $\mathcal{I}_{\mathrm{s}}^{*}$ further ensures that the mapping allows bounding the cost of $\mathcal{G}$ (Lemma 3 (ii)).

Choosing $\mathcal{I}_{\mathrm{s}}^{*}$. We choose the elements of $\mathcal{I}_{\mathrm{s}}^{*}$ according to the following procedure. Initially $\mathcal{I}_{\mathrm{s}}^{*}$ is empty. We consider the intervals of $\mathcal{I}_{\mathrm{s}}$ in decreasing order of their colors, and within each color, in the order of their start points. We add the interval $I$ under consideration to $\mathcal{I}_{\mathrm{s}}^{*}$ if it is not completely contained in the extended hat interval of an interval of $\mathcal{I}_{\mathrm{s}}^{*}$.

Competitiveness of $\mathcal{G}$. We now analyze the properties of $\mathcal{I}_{\mathrm{s}}^{*}$. First we notice that by the way $\mathcal{I}_{\mathrm{s}}^{*}$ is chosen, the union of the extended hat intervals of $\mathcal{I}_{\mathrm{s}}^{*}$ form a contiguous interval which contains the horizon. We next prove the following lemma.

Lemma 2. (i) The hat intervals of the intervals of $\mathcal{I}_{\mathrm{s}}^{*}$ are pairwise disjoint. (ii) For every interval $I_{j} \in \mathcal{I}_{\mathrm{s}}$, there exists an interval $I_{j^{\prime}} \in \mathcal{I}_{\mathrm{s}}^{*}$ such that $I_{j} \subseteq I_{j^{\prime}}^{\mathrm{e}}$ and $\mathcal{G}\left(I_{j}\right) \leq \mathcal{G}\left(I_{j^{\prime}}\right)$.

Proof. (i) Consider any two intervals $I_{j}$ and $I_{j^{\prime}}$ in $\mathcal{I}_{\mathrm{s}}^{*}$. Assume without loss of generality that $I_{j}$ is chosen before $I_{j^{\prime}}$. When $I_{j}$ is chosen, any intervals that are entirely contained in $I_{j}^{\mathrm{e}}$ are removed. Since $I_{j^{\prime}}$ is not removed, at least one of the following conditions holds. (1) $e_{j^{\prime}}>e_{j}+3 k \ell_{\min }$, (2) $s_{j^{\prime}}<s_{j}-3 k \ell_{\min }$. We analyze only the case where (1) holds, the other case being symmetric. If 
(1) holds we have that $s_{j^{\prime}} \geq e_{j^{\prime}}-k \ell_{\min }>e_{j}+2 k \ell_{\min }$ and the left point of $I_{j^{\prime}}^{\mathrm{h}}$ is $s_{j^{\prime}}-k \ell_{\min }>e_{j}+k \ell_{\min }$. Therefore, $I_{j}^{\mathrm{h}}$ and $I_{j^{\prime}}^{\mathrm{h}}$ are disjoint.

(ii) Follows from the way $\mathcal{I}_{\mathrm{s}}^{*}$ is chosen. Consider an interval $I_{j} \in \mathcal{I}_{\mathrm{s}}$. If $I_{j} \in \mathcal{I}_{\mathrm{s}}^{*}$ the claim follows. Otherwise, there is an interval $I_{j^{\prime}} \in \mathcal{I}_{\mathrm{s}}^{*}$ such that $I_{j} \subseteq I_{j^{\prime}}^{\mathrm{e}}$ and $I_{j^{\prime}}$ is considered before $I_{j}$ in the selection process. Therefore, $\mathcal{G}\left(I_{j}\right) \leq \mathcal{G}\left(I_{j^{\prime}}\right)$.

Using Lemma 2, we can relate the cost of the greedy algorithm to the optimum.

Lemma 3. (i) $\operatorname{cost}(\mathcal{O}) \geq \ell_{\min } \cdot \sum_{I \in \mathcal{I}_{\mathrm{s}}^{*}} \mathcal{G}(I)$; (ii) $\operatorname{cost}(\mathcal{G}) \leq 7 k \ell_{\min } \cdot \sum_{I \in \mathcal{I}_{\mathrm{s}}^{*}} \mathcal{G}(I)$; and (iii) $\operatorname{cost}(\mathcal{G}) \leq 7 k \ell(\mathcal{I})$.

Proof. (i) By Lemma 1, for every interval $I \in \mathcal{I}_{\mathrm{s}}^{*}$, there is a set of $\mathcal{G}(I)$ intervals with total length of $\ell_{\min } \mathcal{G}(I)$ each of which is contained in $I^{\mathrm{h}}$. By Lemma 2, the hat intervals of $I \in \mathcal{I}_{\mathrm{s}}^{*}$ are pairwise disjoint. This means the total length of all intervals is at least $\sum_{I \in \mathcal{I}_{\mathrm{s}}^{*}} \ell_{\min } \mathcal{G}(I)$. The statement then follows from Observation 2 .

(ii) Let $\mathcal{I}_{\mathrm{s}}^{\mathrm{e}}$ be the set of extended hat intervals of $\mathcal{I}_{\mathrm{s}}^{*}$, i.e., $\mathcal{I}_{\mathrm{s}}^{\mathrm{e}}=\left\{I^{\mathrm{e}} \mid I \in \mathcal{I}_{\mathrm{s}}^{*}\right\}$, and $\mathcal{G}\left(\mathcal{I}_{\mathrm{s}}^{\mathrm{e}}\right)$ be the coloring of $I^{\mathrm{e}} \in \mathcal{I}_{\mathrm{s}}^{\mathrm{e}}$ using the color of the corresponding interval $I$, i.e., $\mathcal{G}\left(I^{\mathrm{e}}\right)=\mathcal{G}(I)$. Note that $\mathcal{G}$ is not necessarily a valid coloring for $\mathcal{I}_{\mathrm{s}}^{\mathrm{e}}$, but its cost is yet well defined.

By Lemma 2, for every interval $I_{j} \in \mathcal{I}$, there is an interval $I_{j^{\prime}} \in \mathcal{I}_{\mathrm{s}}^{*}$ such that $\mathcal{G}\left(I_{j}\right) \leq \mathcal{G}\left(I_{j^{\prime}}\right)$. If we raise the color of $I_{j}$ from $\mathcal{G}\left(I_{j}\right)$ to $\mathcal{G}\left(I_{j^{\prime}}\right)$, then the resulting skyline is of the same height or higher at every point $t$, in other words, $\operatorname{cost}\left(\mathcal{G}\left(\mathcal{I}_{\mathrm{s}}\right), t\right) \leq \operatorname{cost}\left(\mathcal{G}\left(\mathcal{I}_{\mathrm{s}}^{\mathrm{e}}\right), t\right)$ at every point $t$. Therefore, $\operatorname{cost}(\mathcal{G})=\operatorname{cost}\left(\mathcal{G}\left(\mathcal{I}_{\mathrm{s}}\right)\right) \leq \operatorname{cost}\left(\mathcal{G}\left(\mathcal{I}_{\mathrm{s}}^{\mathrm{e}}\right)\right)$. We also have $\operatorname{cost}\left(\mathcal{G}\left(I^{\mathrm{e}}\right)\right)=\ell\left(I^{\mathrm{e}}\right) \mathcal{G}(I) \leq$ $7 k \ell_{\min } \mathcal{G}(I)$ for every interval $I$. Therefore, $\operatorname{cost}\left(\mathcal{G}\left(\mathcal{I}_{\mathrm{s}}^{\mathrm{e}}\right)\right) \leq 7 k \ell_{\min } \sum_{I \in \mathcal{I}_{\mathrm{s}}^{*}} \mathcal{G}(I)$.

(iii) The proof of (i) states that $\ell(\mathcal{I}) \geq \sum_{I \in \mathcal{I}_{\mathrm{s}}^{*}} \ell_{\min } \mathcal{G}(I)$. Then Statement (ii) implies that $\operatorname{cost}(\mathcal{G}) \leq 7 k \ell(\mathcal{I})$.

Theorem 4. When $\lambda(i)=i$, the greedy algorithm $\mathcal{G}$ is $7 k$-competitive where $k=\ell_{\max } / \ell_{\min } 4^{4}$

\footnotetext{
${ }^{4}$ As was pointed out by an anonymous reviewer of a previous version of this paper, the competitive ratios can be improved to 4 when $k=1$ and 9 when $k=2$ by using a different algorithm, while the ratio becomes $(k+1)^{2}$ for larger $k$. This improvement does not affect the order of the competitive ratio for the general case in Theorem 6 .
} 


\subsection{Arbitrary length intervals}

In this section we consider intervals with arbitrary lengths. We first observe in the following lemma that the greedy algorithm $\mathcal{G}$ performs badly for such instances since $\frac{\ell_{\max }}{\ell_{\min }}$ can be large.

Lemma 4. The greedy algorithm $\mathcal{G}$ is $\Omega\left(\frac{\ell_{\max }}{\ell_{\min }}\right)$-competitive.

Proof. Consider the following instance consisting of $n$ intervals, $I_{j}=[0,1)$ for $j \in[1, n-1]$, and $I_{n}=[0, \ell)$. Consider the coloring $\omega$ such that $\omega\left(I_{n}\right)=1$ and $\omega\left(I_{j}\right)=j+1$ for every $j \in[1, n-1]$. The cost is $\operatorname{cost}(\omega)=(\ell-1)+n$. On the other hand, the greedy algorithm gives the following coloring: $\mathcal{G}\left(I_{j}\right)=j$ for $j \in[1, n-1]$ and $\mathcal{G}\left(I_{n}\right)=n$ and $\operatorname{cost}(\mathcal{G})=n \ell$. We note that the ratio $\frac{\operatorname{cost}(\mathcal{G})}{\operatorname{cost}(\omega)}=\frac{\ell n}{\ell-1+n}$ can be made arbitrarily close to $\ell=\frac{\ell_{\max }}{\ell_{\min }}$.

The greedy algorithm performs badly against the adversary in Lemma 4 because it uses up the small colors for short intervals and then has to use a large color for the long interval. To address this issue, we would like to design an algorithm that distributes the colors among intervals of different lengths in a "fair" way.

In order to obtain a better competitive ratio, we propose the algorithm Classify-greedy which we denote by $\mathcal{C}$. For ease of presentation, we first assume that $\mathcal{C}$ knows in advance $\ell_{\max }$ and $\ell_{\min }$. Let $L=1+\left\lceil\log \frac{\ell_{\max }}{\ell_{\min }}\right\rceil$. We partition $\mathcal{I}$ into $L$ classes $C_{1}, C_{2}, \cdots, C_{L}$ such that $C_{i}$ contains all intervals $I$ with $\ell(I) \in\left[\ell_{\min } \cdot 2^{i-1}, \ell_{\min } \cdot 2^{i}\right)$. Furthermore, we also partition the set of colors $\Lambda$ into $L$ disjoint sets, where $\Lambda_{i}=\{i, i+L, i+2 L, i+3 L, \cdots\}$, for $i \in[L]$.

Classify-greedy $\mathcal{C}$ runs $L$ copies $\mathcal{G}_{1}, \ldots \mathcal{G}_{L}$ of $\mathcal{G}$ where $\mathcal{G}_{i}$ uses the set of colors $\Lambda_{i}$. When $I \in C_{i}$ arrives, it is processed by $\mathcal{G}_{i}$ which colors it with the smallest color in $\Lambda_{i}$ that is valid for $I$.

We denote an optimal coloring of $\mathcal{I} \cap C_{i}$ by $\mathcal{O}_{i}$. The following observation is due to Lemma 3(iii) (for $k=2$ ) and the fact that $\mathcal{G}_{i}$ uses $\Lambda_{i}$ that contains one color per every interval of $L$ colors.

Observation 5. $\operatorname{cost}\left(\mathcal{G}_{i}\right) \leq 14 L \cdot \ell\left(C_{i}\right)$.

Theorem 6. Algorithm $\mathcal{C}$ is $O\left(\left\lceil\log \frac{\ell_{\max }}{\ell_{\min }}\right\rceil\right)$-competitive. 
Proof. The cost of $\mathcal{C}$ is the integral over the horizon of the maximum color used by all copies of $\mathcal{G}$ at every point $t$, i.e.,

$$
\begin{aligned}
\operatorname{cost}(\mathcal{C}) & =\int_{-\infty}^{\infty} \max _{i \in[L]} \cos t\left(\mathcal{G}_{i}, t\right) \mathrm{d} t \leq \int_{-\infty}^{\infty} \sum_{i=1}^{L} \operatorname{cost}\left(\mathcal{G}_{i}, t\right) \mathrm{d} t \\
& =\sum_{i=1}^{L} \int_{-\infty}^{\infty} \operatorname{cost}\left(\mathcal{G}_{i}, t\right) \mathrm{d} t=\sum_{i=1}^{L} \operatorname{cost}\left(\mathcal{G}_{i}\right) \leq \sum_{i=1}^{L} 14 L \cdot \ell\left(C_{i}\right) \\
& =14 L \cdot \ell(\mathcal{I}) \leq 14 L \cdot \mathcal{O} .
\end{aligned}
$$

Knowing the ratio $\frac{\ell_{\max }}{\ell_{\min }}$ only. We now describe how the algorithm can be adapted to the setting where only the ratio $\frac{\ell_{\max }}{\ell_{\min }}$ is known instead of knowing $\ell_{\max }$ and $\ell_{\min }$. An interval of length $\ell$ is assigned to the class $\left\lceil\log _{2} \ell\right\rceil$. In this way, the intervals are assigned to at most $L+1$ length classes with consecutive indices though the indices may not be from 1 to $L+1$. The set of colors $\Lambda$ is now divided into $L+1$ disjoint sets $\Lambda_{1}, \Lambda_{2}, \cdots, \Lambda_{L+1}$ (recall that $\left.\Lambda_{i}=\{i, i+L+1, i+2(L+1), i+3(L+1), \cdots\}\right)$. When an interval in a new length class is released, we map this length class to the next available color set. We note that Observation 5 remains correct with $L=2+\left\lceil\log \frac{\ell_{\max }}{\ell_{\min }}\right\rceil$ and Theorem 6 follows with competitive ratio $14\left(2+\left\lceil\log \frac{\ell_{\max }}{\ell_{\min }}\right\rceil\right)$, which is still $O\left(\left\lceil\log \frac{\ell_{\max }}{\ell_{\min }}\right\rceil\right)$.

\subsection{Lower bound}

In this section, we present an adversary to show a lower bound for any deterministic online algorithm that asymptotically matches the upper bound shown in Section 4.2 for Classify-greedy.

Theorem 7. No deterministic online algorithm can achieve competitive ratio better than $\frac{1}{2} \log \frac{\ell_{\max }}{\ell_{\min }}$ even if it knows $\frac{\ell_{\max }}{\ell_{\min }}$ in advance. This holds even when the intervals are released from left to right and even for special instances including proper instances and laminar instances. 55

\footnotetext{
${ }^{5} \mathrm{An}$ instance is a proper instance if for any two intervals $I_{1}$ and $I_{2}, s_{1} \leq s_{2}$ implies $e_{1} \leq e_{2}$. An instance is a laminar instance if any two intervals are either disjoint or one is completely contained in another.
} 
Proof. Let $\mathcal{A}$ be an online algorithm. Let $L$ be an arbitrarily large positive integer. The adversary creates an instance $\mathcal{I}$ with $\frac{\ell_{\max }}{\ell_{\min }}=2^{L}$, or equivalently $\log \frac{\ell_{\max }}{\ell_{\min }}=L$, as follows. We first present the construction of a laminar instance. The instance will be such that it is easy to see that a load-optimal coloring exists.

The adversary releases a sequence of up to $L$ intervals $I_{j}=\left[0,2^{j}\right)$ for $j=1,2, \ldots, L$. If the algorithm uses color $L+1$ for one of them, say for interval $I_{k}$, the adversary stops the sequence and presents only one more final interval $I_{f}=\left[2^{k}, 2^{k}+\frac{1}{2^{L-k}}\right)$. Note that $\ell_{\max }=2^{k}$ and $\ell_{\min }=2^{-(L-k)}$, so $\frac{\ell_{\max }}{\ell_{\min }}=2^{L}$. We have $\operatorname{cost}(\mathcal{A}) \geq(L+1) 2^{k}$ and $\operatorname{cost}(\mathcal{O})=\ell(\mathcal{I})<2^{k+1}$, so $\frac{\operatorname{cost}(\mathcal{A})}{\operatorname{cost}(\mathcal{O})}>\frac{L+1}{2}>\frac{L}{2}$.

If the algorithm does not use color $L+1$ on the $L$ intervals of the sequence, it must use colors $1, \ldots, L$ on these intervals as they all overlap. The adversary then presents one more interval $I_{L+1}=\left[0,2^{L+1}\right)$, which must receive color at least $L+1$. Note that $\ell_{\max }=2^{L+1}$ and $\ell_{\min }=2$, so $\frac{\ell_{\max }}{\ell_{\min }}=2^{L}$. We have $\operatorname{cost}(\mathcal{A}) \geq(L+1) 2^{L+1}$ and $\operatorname{cost}(\mathcal{O})=\ell(\mathcal{I}) \leq 2^{L+2}$, so $\frac{\operatorname{cost}(\mathcal{A})}{\operatorname{cost}(\mathcal{O})} \geq \frac{L+1}{2}>\frac{L}{2}$.

The above instance is a laminar instance. We can change the construction to produce a proper instance by a slight modification: Let $\epsilon$ be a very small positive value; then $I_{j}$ is set to $\left[(j-1) \epsilon, 2^{j}+(j-1) \epsilon\right)$ for $1 \leq j \leq L+1$, and $I_{f}$ is set to $\left[2^{k}+(k-1) \epsilon, 2^{k}+\frac{1}{2^{L-k}}+(k-1) \epsilon\right)$. This instance does not have a load-optimal coloring, but the cost of an optimal coloring is larger than the total length of the set of intervals by less than $\left(L^{2}+L\right) \epsilon$ (note that the only part of the line in which the maximum color index used is larger than the load lies between 0 and $L \epsilon$, and the maximum color used is $L+1$ ), which is negligible for small enough $\epsilon$. Therefore, the analysis of the laminar case applies also here.

In both cases, intervals are released from left to right.

\section{Extensions}

\subsection{Uniform color capacity}

We consider the extension where each color has a "capacity" $\kappa$ : it is allowed to have $\kappa$ overlapping intervals sharing the same color at the same point. A coloring $\omega: \mathcal{I} \rightarrow \Lambda$ is valid if for any $c \in \Lambda$ and at any point $t$, there are at most $\kappa$ intervals $I \in \mathcal{I}_{t}$ with $\omega(I)=c$. In this case, we show that we can adapt the algorithms in Section 4 with a constant factor increase in the competitive ratio. 
Adapted algorithms. First, we observe that Observation 2 can be adapted to $\operatorname{cost}(\omega) \geq \frac{\ell(\mathcal{I})}{\kappa}$ because there can be at most $\kappa$ intervals sharing a color at any point $t$, i.e., $\operatorname{cost}(\omega, t) \geq\left\lceil\frac{\operatorname{load}(t)}{\kappa}\right\rceil$, and $\int_{-\infty}^{\infty} \operatorname{load}(t) \mathrm{d} t=\ell(\mathcal{I})$. The color assignment of the greedy algorithm $\mathcal{G}$ remains the same except that the condition of valid coloring is now adapted as above to allow $\kappa$ intervals sharing a color. Then the algorithm Classify-greedy $\mathcal{C}$ is exactly the same as before, but using the adapted $\mathcal{G}$. The analysis is also similar but more involved.

Adapted analysis. In the analysis of $\mathcal{G}$, we rely on the fact that when $\mathcal{G}$ assigns color $c$ to an interval $I_{j}$, there are a substantial number of intervals overlapping with $I_{j}$ in the input (see Lemma 1). With the capacity, we show a variant of Lemma 1: the length of $I_{j}$ plus the total length of the $(c-1) \kappa$ intervals that overlap with $I_{j}$ and are contained in $I_{j}^{\mathrm{h}}$ is at least $((c-1) \kappa+1) \cdot \ell_{\min }$ (see Lemma 5). The main difference of this property is that we are no longer able to show that the total length of overlapping intervals is $c \kappa \ell_{\min }$, i.e., we have $(c-1) \kappa \ell_{\min }$ instead of the desired $c \kappa \ell_{\min }$. Recall that the analysis uses a charging scheme that maps intervals on the skyline to certain intervals in the optimal coloring. Most of the analysis still carries forward except for intervals that are colored with color 1 because in such case the new bound only guarantees that intervals with a total length of $\ell_{\min }$ overlap with such an interval (instead of the desired $\kappa \ell_{\text {min }}$ ). Yet for intervals that are colored with color 1, this means that the optimal algorithm also needs to use at least color 1 because there is indeed an interval.

Roughly speaking, we divide the analysis into two parts: (i) $\mathcal{I}_{\mathrm{s}}^{*}$ is selected from highest color until color 2 (instead of color 1) with the same analysis as before and (ii) the remaining skyline intervals colored with color 1 are compared directly to the optimal coloring.

Let $\mathcal{T}$ denote the whole horizon of points.

Observation 8. (Adapted Observation 2) For every valid coloring $\omega$ of a set $\mathcal{I}$ of intervals, we have $\operatorname{cost}(\omega) \geq \frac{\ell(\mathcal{I})}{\kappa}$ when $\lambda(i)=i$ for all $i$.

Lemma 5. (Adapted Lemma 1) Consider an interval $I_{j}$ with $\mathcal{G}\left(I_{j}\right)=c$. (i) There are at least $(c-1) \kappa$ intervals that overlap with $I_{j}$ and are contained in $I_{j}^{\mathrm{h}}$; (ii) the total length of these $(c-1) \kappa$ intervals and $I_{j}$ is at least $((c-1) \kappa+1) \cdot \ell_{\min }$.

Proof. (i) Since $\mathcal{G}$ assigns the smallest possible color to any interval, $I_{j}$ gets color $c$ only if there are already $\kappa$ intervals colored by $1, \kappa$ intervals colored by 
$2, \cdots, \kappa$ intervals colored by $c-1$ and all overlap with $I_{j}$. Since the length of any interval is bounded by $k \ell_{\min }$, for each of these intervals, its start point is at least $s_{j}-k \ell_{\min }$ and its end point is at most $e+k \ell_{\min }$, meaning that they are all inside $I_{j}^{\mathrm{h}}$. (ii) (Same as the original one.)

The selection of skyline intervals is the same as the original one except that we stop before including skyline intervals with color 1 . The horizon $\mathcal{T}$ is partitioned into two parts, $\mathcal{T}^{\prime}$ and $\mathcal{T} \backslash \mathcal{T}^{\prime}$, where $\mathcal{T}^{\prime}$ is the union of the extended hat intervals of all skyline intervals. Note that for all $t \in \mathcal{T} \backslash \mathcal{T}^{\prime}$, $\operatorname{cost}(\mathcal{G}, t)=1$. Also, for each skyline interval $I, \mathcal{G}(I) \geq 2$.

Lemma 6. (Adapted Lemma 3) (i) $\operatorname{cost}\left(\mathcal{G}\left(\mathcal{T}^{\prime}\right)\right) \leq 7 k \ell_{\min } \cdot \sum_{I \in \mathcal{I}_{\mathbf{s}}^{*}} \mathcal{G}(I)$; (ii) $\ell(\mathcal{I}) \geq \ell_{\min } \cdot \kappa \cdot \sum_{I \in \mathcal{I}_{s}^{*}}(\mathcal{G}(I)-1)$; (iii) $\operatorname{cost}\left(\mathcal{G}\left(\mathcal{T} \backslash \mathcal{T}^{\prime}\right)\right) \leq \operatorname{cost}(\mathcal{O})$; and (iv) $\operatorname{cost}\left(\mathcal{G}\left(\mathcal{T}^{\prime}\right)\right) \leq 14 k \cdot \frac{\ell(\mathcal{I})}{\kappa}$.

Proof. (i) (Same as the proof of Lemma 3 (i).) (ii) By Lemma 5, $\ell(\mathcal{I}) \geq$ $\sum_{I \in \mathcal{I}_{\mathrm{s}}^{*}} \ell_{\min } \cdot \kappa \cdot(\mathcal{G}(I)-1)$. (iii) It is trivial. (iv) By (i) and (ii), $\operatorname{cost}\left(\mathcal{G}\left(\mathcal{T}^{\prime}\right)\right) \leq$ $7 k \ell_{\min } \cdot \sum_{I \in \mathcal{I}_{s}^{*}} \mathcal{G}(I) \leq 7 k \sum_{I \in \mathcal{I}_{s}^{*}} \mathcal{G}(I) \cdot \frac{\ell(\mathcal{I})}{\kappa \cdot \sum_{I \in \mathcal{I}_{s}^{*}}(\mathcal{G}(I)-1)}=7 k \cdot \frac{\ell(\mathcal{I})}{\kappa} \cdot \frac{\sum_{I \in \mathcal{I}_{s}^{*}} \mathcal{G}(I)}{\sum_{I \in \mathcal{I}_{s}^{*}}(\mathcal{G}(I)-1)}$. Since $\mathcal{G}(I) \geq 2$ for any $I \in \mathcal{I}_{\mathrm{s}}^{*}, \frac{\sum_{I \in \mathcal{I}_{\mathrm{s}}^{*} \mathcal{G}(I)}}{\sum_{I \in \mathcal{I}_{\mathrm{s}}^{*}}(\mathcal{G}(I)-1)} \leq 2$. Hence, $\operatorname{cost}\left(\mathcal{G}\left(\mathcal{T}^{\prime}\right)\right) \leq$ $14 k \cdot \frac{\ell(\mathcal{I})}{\kappa}$.

Theorem 9. (Adapted Theorem 4) When $\lambda(i)=i$, the greedy algorithm $\mathcal{G}$ is $\left(14 \ell_{\max } / \ell_{\min }+1\right)$-competitive for the uniform color capacity setting.

Proof. By definition, $\operatorname{cost}(\mathcal{G})=\operatorname{cost}\left(\mathcal{G}\left(\mathcal{T}^{\prime}\right)\right)+\operatorname{cost}\left(\mathcal{G}\left(\mathcal{T} \backslash \mathcal{T}^{\prime}\right)\right)$. By Lemma 6 (iv) and Observation $8, \operatorname{cost}\left(\mathcal{G}\left(\mathcal{T}^{\prime}\right)\right) \leq 14 k \cdot \operatorname{cost}(\mathcal{O})$. By Lemma 6 (iii), $\operatorname{cost}(\mathcal{G})=\operatorname{cost}\left(\mathcal{G}\left(\mathcal{T}^{\prime}\right)\right)+\operatorname{cost}\left(\mathcal{G}\left(\mathcal{T} \backslash \mathcal{T}^{\prime}\right)\right) \leq 14 k \cdot \operatorname{cost}(\mathcal{O})+\operatorname{cost}(\mathcal{O})$.

Similarly, the analysis of $\mathcal{C}$ also takes the approach of dividing the horizon into two parts: those with intervals colored higher than color $L$ and those with intervals colored $L$ or lower; the latter corresponds to color 1 for each length class. More precisely, the horizon $\mathcal{T}$ is partitioned into two parts, $\mathcal{T}^{\prime \prime}$ and $\mathcal{T} \backslash \mathcal{T}^{\prime \prime}$. A point $t$ is in $\mathcal{T}^{\prime \prime}$ if there is at least one interval $I$ such that $t \in I^{\mathrm{e}}$ and $\mathcal{G}(I)>L$. Letting $\mathcal{T}_{i}^{\prime \prime}$ denote $\mathcal{T}^{\prime \prime}$ defined with respect to $\mathcal{G}_{i}\left(C_{i}\right)$, we have $\mathcal{T}^{\prime \prime}=\cup_{i} \mathcal{T}_{i}^{\prime \prime}$.

Observation 10. (Adapted Observation $5 \operatorname{cost}\left(\mathcal{G}_{i}\left(\mathcal{T}_{i}^{\prime \prime}\right)\right) \leq 28 L \cdot \frac{\ell\left(C_{i}\right)}{\kappa}$. 
Theorem 11. (Adapted Theorem 6) Algorithm $\mathcal{C}$ is $O\left(\left\lceil\log \frac{\ell_{\max }}{\ell_{\min }}\right\rceil\right)$-competitive for the uniform color capacity setting.

Proof. As we have shown in Theorem 6 , the cost of $\mathcal{C}$ is at most the integral over the horizon of the maximum color used by all copies of $\mathcal{G}$ at every point $t$, i.e., $\cos t(\mathcal{C}) \leq \int_{-\infty}^{\infty} \max _{i \in[L]} \cos t\left(\mathcal{G}_{i}, t\right) \mathrm{d} t$. As we partition the horizon, the latter is equivalent to $\int_{\mathcal{T}^{\prime \prime}} \max _{i \in[L]} \operatorname{cost}\left(\mathcal{G}_{i}, t\right) \mathrm{d} t+\int_{\mathcal{T} \backslash \mathcal{T}^{\prime \prime}} \max _{i \in[L]} \operatorname{cost}\left(\mathcal{G}_{i}, t\right) \mathrm{d} t$. Similar to the proof of Theorem 6, we have $\int_{\mathcal{T}^{\prime \prime}} \max _{i \in[L]} \operatorname{cost}\left(\mathcal{G}_{i}, t\right) \mathrm{d} t \leq$ $\sum_{i=1}^{L} \operatorname{cost}\left(\mathcal{G}_{i}\left(\mathcal{T}_{i}^{\prime \prime}\right)\right) \leq \sum_{i=1}^{L} 28 L \cdot \frac{\ell\left(C_{i}\right)}{\kappa}$. The latter inequality is due to Observation 10. We also note that $\sum_{i=1}^{L} \frac{\ell\left(C_{i}\right)}{\kappa}=\frac{\ell(\mathcal{I})}{\kappa} \leq \operatorname{cost}(\mathcal{O})$ because of Observation 8. On the other hand, $\int_{\mathcal{T} \backslash \mathcal{T}^{\prime \prime}} \max _{i \in[L]} \operatorname{cost}\left(\mathcal{G}_{i}, t\right) \leq \int_{\mathcal{T} \backslash \mathcal{T}^{\prime \prime}} L \mathrm{~d} t \leq$ $L \cdot \operatorname{cost}(\mathcal{O})$. Combining the two results, we have $\operatorname{cost}(\mathcal{C}) \leq 29 L \cdot \operatorname{cost}(\mathcal{O})$.

\subsection{Generalized color cost function}

A more general problem of SKYLINE is to generalize the cost function of colors. In the original SKYLINE problem, we assume that $\lambda(i)=i$ for all colors $i \in \Lambda$. We relax this constraint by considering a bounded relative cost of the neighboring colors, i.e., $1 \leq \frac{\lambda(i+1)}{\lambda(i)} \leq \delta$. Note that for the original

setting we have $\frac{\lambda(i+1)}{\lambda(i)} \leq 2$. The SKYLINE problem under this new setting, however, is much harder in the sense of competitive ratio. In fact, we show in Theorem 12 that the lower bound on the competitive ratio of this problem is exponential in $n$. On the other hand, the competitive ratio in terms of $n$ for the original setting can be shown to be $\Theta(n)$ as follows. The greedy algorithm is $O(n)$-competitive because it colors any interval by a color at most $n$ and so the cost of the greedy algorithm is at most $n \cdot h$ and the optimal cost is at least $h$, where $h$ is the length of the horizon. A lower bound of $\Omega(n)$ follows from the construction in the proof of Theorem 7 since the number of intervals in the construction is at most $L+1$.

Theorem 12. Consider SKYLINE. For every $\delta>1$, there exists a cost function $\lambda$ such that $\frac{\lambda(i+1)}{\lambda(i)} \leq \delta$ for all $i \geq 1$ and $\frac{\operatorname{cost}(\mathcal{A})}{\operatorname{cost}(\mathcal{O})} \geq \delta^{\Omega(n)}$.

Proof. We consider the cost function $\lambda(i)=\delta^{i-1}$. Note that $\frac{\lambda(i+1)}{\lambda(i)}=\delta$. We apply an adversary that is similar to the one in the proof of Theorem 7 . The adversary creates an instance $\mathcal{I}$ such that it is easy to see that a load-optimal coloring exists. Let $\mathcal{A}$ be an online algorithm, $L$ be an arbitrarily large 
positive integer and $d=\lceil\delta\rceil+1$. The adversary releases a sequence of up to $L$ intervals $I_{j}=\left[0, d^{j}\right)$ for $j=1,2, \ldots, L$.

Considering the set of the $x$ intervals $I_{j}=\left[0, d^{j}\right)$ for $1 \leq j \leq x$, the optimal cost is at most $d^{x+1}$ due to the following.

$$
\begin{aligned}
\operatorname{cost}(\mathcal{O}) & =d \cdot \delta^{x-1}+\sum_{i=1}^{x-1}\left(d^{i+1}-d^{i}\right) \cdot \delta^{x-(i+1)} \\
& =d \cdot \delta^{x-1}+(d-1) \cdot\left(\delta \cdot d^{x}-d \cdot \delta^{x}\right) /(\delta(d-\delta)) \\
& \leq d \cdot \delta^{x-1}+(d-1) \cdot d^{x} \leq d^{x+1}
\end{aligned}
$$

The first equality holds because in the optimal solution the interval $I_{i}$ is assigned color $x+1-i$ with cost $\lambda(x-i)=\delta^{x-i}$ for all $1 \leq i \leq x$, and each interval $I_{i+1}$ of length $d^{i+1}$, for $1 \leq i \leq x-1$, contributes a part of length $d^{i+1}-d^{i}$ (with cost $\delta^{x-(i+1)}$ at each point) to the skyline. The first inequality is due to $d-\delta \geq 1$ and the second inequality is because $\delta \leq d-1$.

If the algorithm uses color $L+1$ for one of the released intervals, say for interval $I_{k}$, the adversary stops the sequence. We have $\operatorname{cost}(\mathcal{A}) \geq d^{k} \cdot \delta^{L}$ and $\operatorname{cost}(\mathcal{O}) \leq d^{k+1}$, so $\frac{\operatorname{cost}(\mathcal{A})}{\operatorname{cost}(\mathcal{O})} \geq \frac{\delta^{L}}{d} \geq \frac{\delta^{L}}{3 \delta}=\frac{\delta^{L-1}}{3}$. Note that $d \leq 3 \delta$ since $\delta>1$.

If the algorithm does not use color $L+1$ on the $L$ intervals of the sequence, it must use colors $1, \ldots, L$ on these intervals as they all overlap. The adversary then presents one more interval $I_{L+1}=\left[0, d^{L+1}\right)$, which must receive color at least $L+1$. We have $\operatorname{cost}(\mathcal{A}) \geq d^{L+1} \cdot \delta^{L}$ and $\operatorname{cost}(\mathcal{O}) \leq d^{L+2}$, so $\frac{\operatorname{cost}(\mathcal{A})}{\operatorname{cost}(\mathcal{O})} \geq \frac{\delta^{L}}{d} \geq \frac{\delta^{L}}{3 \delta}=\frac{\delta^{L-1}}{3}$.

\subsection{Circular graphs}

The upper and lower bound results in Section 4 apply to circular graphs as well. For the lower bound this is obvious. For the upper bound, suppose we have a circle running clockwise from label 0 until label $\mathcal{T}(0$ coincides with $\mathcal{T})$. An input interval consists of a start point and an end point. If the start point has label larger than the end point, this means the interval runs across point 0 . Without loss of generality, we assume that the union of the input intervals cover the entire circle, otherwise, the input can be treated as an input on a line. The algorithm $\mathcal{G}$ works the same way on a circle. The analysis needs modification for intervals crossing the point 0 . For an interval $[s, e)$, the hat and extended hat interval is now defined as $\left[\left(s_{j}-k \ell_{\min }\right) \bmod \mathcal{T},\left(e_{j}+k \ell_{\min }\right)\right.$ $\bmod \mathcal{T})$ and $\left[\left(s_{j}-3 k \ell_{\min }\right) \bmod \mathcal{T},\left(e_{j}+3 k \ell_{\min }\right) \bmod \mathcal{T}\right)$, respectively. With this definition, we select $\mathcal{I}_{\mathrm{s}}^{*}$ the same way as before until the whole horizon 
is covered by the span of the extended hat intervals of selected intervals. In this way, Lemma 2 remains correct and the analysis follows.

\section{The Permutation problem}

In this section, we consider a variant of the SKYLINE problem. Throughout this section, we assume $\lambda(i)=i$ for all $i$. A solution of the SKYLINE problem can be obtained by first partitioning $\mathcal{I}$ into disjoint subsets such that the intervals of every subset are pairwise disjoint, and then assigning distinct colors to the subsets. The second stage is exactly the problem of finding a permutation of the subsets of intervals.

Precisely, we define the problem Permutation as follows. We are given $|\Lambda|$ disjoint sets of intervals $\mathcal{I}_{1}, \mathcal{I}_{2}, \cdots, \mathcal{I}_{|\Lambda|}$ such that the intervals in each set are pairwise disjoint, i.e., all the intervals of a set $\mathcal{I}_{i}$ can be assigned the same color. The goal is to find a permutation $\pi$ of the colors such that $\mathcal{I}_{i}$ is assigned the color $\pi(i)$ and the total cost of the coloring induced by $\pi$ is minimized.

At first glance, the permutation problem may look simpler since the partition into sets is already given. Yet we show in Section 6.1 that the permutation problem is NP-hard. Note that there is no requirement that the given partition has a permutation that is an optimal solution to SKYLINE. Our NP-hardness proof does not imply anything regarding the complexity of $\pi$ when it has a solution that is optimal for SKYLINE. We propose a 2-approximation algorithm in Section 6.2.

\subsection{NP-hardness}

The NP-hardness proof is by reduction from the optimal linear arrangement problem.

Optimal linear arrangement (LINA). The input is a graph $G=(V, E)$, and the goal is to find a one-to-one function $f: V \rightarrow\{1,2, \ldots,|V|\}$ such that $\sum_{(u, v) \in E}|f(v)-f(u)|$ is minimized. The decision version of this problem is known to be NP-hard (see [9]).

We denote the degree of a vertex $v \in V$ by $d(v)$. We also denote the maximum degree of all vertices by $\Delta$.

The reduction. Given a simple graph $G=(V, E)$ which is an instance of Lina, we construct an instance $\mathcal{I}$ of Permutation. For each vertex $v \in V$, we create a subset of intervals $\mathcal{I}_{v} \subseteq \mathcal{I}$ such that the intervals in $\mathcal{I}_{v}$ are pairwise disjoint. The details of the construction are as follows. For each edge 
$e=u v \in E$, we create an edge gadget containing two identical intervals $I_{u}^{e}$ and $I_{v}^{e}$ of length 2. The intervals corresponding to distinct edges are disjoint. Then for every vertex $v \in V$, we create a dummy interval of length $\Delta-d(v)$. Each dummy interval is disjoint from any other interval in the construction. Overall, the set $\mathcal{I}_{v}$ consists of all intervals $I_{v}^{e}$ where $v$ is an endpoint of edge $e$ and its dummy interval. The input $\mathcal{I}$ is then $\left\{\mathcal{I}_{v} \mid v \in V\right\}$. We are going to prove the following theorem.

Theorem 13. The Permutation problem is NP-hard.

Proof. Consider a solution $\pi$ of Permutation on instance $\mathcal{I}$. The cost of the two intervals associated with an edge $e=u v$ is

$$
2 \max \{\pi(u), \pi(v)\}=\pi(u)+\pi(v)+|\pi(u)-\pi(v)| .
$$

The cost of a dummy interval associated with a vertex $v$ is

$$
(\Delta-d(v)) \pi(v)
$$

Summing up the first cost over all edges and the second over all vertices we get the following expression for the cost of $\pi$.

$\operatorname{Permutation}(\mathcal{I}, \pi)$

$$
\begin{aligned}
& =\sum_{e=u v \in E}(\pi(u)+\pi(v))+\sum_{e=u v \in E}|\pi(u)-\pi(v)|+\sum_{v \in V}(\Delta-d(v)) \pi(v) \\
& =\operatorname{LiNA}(G, \pi)+\sum_{e=u v \in E}(\pi(u)+\pi(v))+\sum_{v \in V}(\Delta-d(v)) \pi(v) \\
& =\operatorname{LiNA}(G, \pi)+\sum_{v \in V} \pi(v) d(v)+\sum_{v \in V}(\Delta-d(v)) \pi(v) \\
& =\operatorname{LiNA}(G, \pi)+\sum_{v \in V} \Delta \cdot \pi(v)=\operatorname{LiNA}(G, \pi)+\Delta \sum_{v \in V} \pi(v) \\
& =\operatorname{LiNA}(G, \pi)+\frac{\Delta \cdot|V| \cdot(|V|+1)}{2} .
\end{aligned}
$$

Since the second term does not depend on $\pi$, minimizing Permutation $(\mathcal{I}, \pi)$ is equivalent to minimizing $\operatorname{LinA}(G, \pi)$. 


\subsection{Approximation algorithm}

We design a 2-approximation algorithm for the permutation problem by using a linear programming relaxation and show that the analysis is tight by proving that the integrality gap of the linear program is 2 .

The permutation problem can be considered as a matching problem that matches colors to sets of intervals. Let $m_{j, i}=1$ indicate that the $j$-th color is matched to the $i$-th set of intervals and $m_{j, i}=0$ otherwise. The permutation problem thus aims to determine $m_{j, i}$ for all $j$ and $i$ such that the total cost is minimized. Without loss of generality, we assume that the horizon is divided into $t$ segments of unit length such that all intervals start and end at the boundaries of these segments, i.e. no interval starts or ends in the interior of a segment. Although the number of unit segments $t$ can be arbitrarily large, we can remove this assumption by combining adjacent unit segments such that for each combined segment, both its left boundary and right boundary are the start or end points of at least one interval. The cost of this combined segment is the skyline of this segment times the length of this segment. The number of combined segments is at most $2 n-1$, which allows the following relaxed linear program to be constructed and solved in polynomial time. For ease of presentation, we present the linear program for the unit segments instead of the combined segments.

By considering the matching problem and the unit segments, we can write a linear program for PERMUtATION. Let $a_{i, u}=1$ indicate that the $i$-th set of intervals covers the $u$-th unit segment, i.e. that the $u$-th unit segment is entirely covered by some interval in the $i$-th set of intervals, and $a_{i, u}=0$ otherwise. Let also $s_{u}$ be the skyline of the $u$-th unit segment. The integer linear program that models the PERMUTATION problem is as follows.

$$
\text { Minimize } \sum_{u=1}^{t} s_{u}
$$


subject to

$$
\begin{array}{cr}
\sum_{i=1}^{|\Lambda|} m_{j, i}=1 & \forall j \in[|\Lambda|] \\
\sum_{j=1}^{|\Lambda|} m_{j, i}=1 & \forall i \in[|\Lambda|] \\
a_{i, u} \cdot \sum_{j=1}^{|\Lambda|} j \cdot m_{j, i} \leq s_{u} & \forall i \in[|\Lambda|], u \in[t] \\
m_{j, i} \in\{0,1\} & \forall i, j \in[|\Lambda|]
\end{array}
$$

Our algorithm solves the relaxed version of the above program that is obtained by replacing the integrality constraints (4) by constraints (5), and matches the colors with the sets of intervals based on the computed solution.

$$
m_{j, i} \in[0,1] \quad \forall i, j \in[|\Lambda|]
$$

Algorithm $\mathcal{B}$. Let $c_{i}=\sum_{j=1}^{|\Lambda|} j \cdot m_{j, i}$ be the estimated color of the $i$-th set of intervals provided by the linear program, for every $i \in[|\Lambda|]$. $\mathcal{B}$ solves the relaxed linear program and obtains all $c_{i}$ 's. We denote by $c^{(k)}$ the $k$-th smallest number in $\left\{c_{1}, c_{2}, \ldots, c_{|\Lambda|}\right\}$ with ties broken arbitrarily. Then $\mathcal{B}$ assigns to the $i$-th set of intervals the color $k$ such that $c_{i}=c^{(k)}$.

Theorem 14. Algorithm $\mathcal{B}$ is 2-approximation for PERMUTATION.

We argue in the following that for every unit length segment $u \in[t]$, its skyline returned by $\mathcal{B}$ is at most twice its skyline $s_{u}$ given by the solution of the relaxed linear program. Thus Theorem 14 follows.

Let $u \in[t]$ be any unit length segment. Note that given an optimal solution of the relaxed linear program, there is at least one $i \in[|\Lambda|]$ such that the corresponding contraint (3) is satisfied with equality. This is because, otherwise one can obtain a super-optimal solution by replacing the value of $s_{u}$ with a smaller value. Furthermore, for this value of $i$, we have $a_{i, u}=1$. We conclude that $s_{u}=\max \left\{c_{i} \mid a_{i, u}=1, i \in[|\Lambda|]\right\}$. The behavior of $\mathcal{B}$ is such that if $s_{u}=c^{(k)}$, then the skyline of $u$ returned by $\mathcal{B}$ is $k$.

We show in the following that the sum of the smallest $k$ values $c^{(1)}, \ldots, c^{(k)}$ is at least $\frac{k(k+1)}{2}$. Therefore, the maximum of these values, i.e. $c^{(k)}$ is at least 
their average, i.e. $\frac{k+1}{2}$, implying $k<k+1 \leq 2 c^{(k)}$. Therefore, the skyline $k$ of $u$ returned by $\mathcal{B}$ is at most twice its skyline $s_{u}$ in the relaxed solution, as required.

\section{Lemma 7.}

$$
\sum_{k=1}^{x} c^{(k)} \geq \frac{x(x+1)}{2} \quad \forall 1 \leq x \leq|\Lambda| .
$$

Proof. Constraints (1), (2) and (5) imply that the matrix $M=\left[m_{j, i}\right](i, j \in$ $[|\Lambda|])$ is doubly stochastic. Let $m$ be a matching that minimizes $\sum_{k=1}^{x} c^{(k)}$ among all matchings, and assume without loss of generality that sets of intervals are ordered by their estimated colors in $m$, i.e. $c_{1} \leq \ldots \leq c_{|\Lambda|}$. We write $M=\left[\begin{array}{ll}M_{1} & M_{2} \\ M_{3} & M_{4}\end{array}\right]$ where $M_{1}$ is the $x$ by $x$ submatrix $M_{1}=\left[m_{j, i}\right](i, j \in$ $[x])$. We claim that at least one of $M_{2}, M_{3}$ is zero. Suppose for a contradiction that both of $M_{2}$ and $M_{3}$ are non-zero, and let $m_{j, i}$ (resp. $m_{j^{\prime}, i^{\prime}}$ ) be a non-zero entry of $M_{2}\left(\operatorname{resp} . M_{3}\right)$. Let also $\varepsilon=\min \left\{m_{j, i}, m_{j^{\prime}, i^{\prime}}\right\}$. We now consider a modified matching $m^{\prime}$ and the corresponding matrix $M^{\prime}$ whose entries are equal to the entries of $M$ except the following four entries that are different: $m_{j, i}^{\prime}=m_{j, i}-\varepsilon, m_{j^{\prime}, i^{\prime}}^{\prime}=m_{j^{\prime}, i^{\prime}}-\varepsilon, m_{j, i^{\prime}}^{\prime}=m_{j, i^{\prime}}+\varepsilon, m_{j^{\prime}, i}^{\prime}=m_{j^{\prime}, i}+\varepsilon$. See Figure 3 for an example. Clearly, $M^{\prime}$ is doubly stochastic. Furthermore, the estimated color of every set of intervals is equal in $m$ and $m^{\prime}$ except for the set of intervals $i>x$ (resp. $\left.i^{\prime} \leq x\right)$ whose estimated color is higher (resp. lower) in $m^{\prime}$ by $\varepsilon\left(j^{\prime}-j\right)$. Therefore, the interval sets with the smallest $x$ estimated colors are the same in $m$ and $m^{\prime}$. This also implies that $\sum_{k=1}^{x} c^{\prime(k)}<\sum_{k=1}^{x} c^{(k)}$ (where $c^{\prime(k)}$ is the $k$-th smallest estimated color in $m^{\prime}$ ), which contradicts the assumption that $m$ minimizes $\sum_{k=1}^{x} c^{(k)}$. Therefore, either $M_{2}$ or $M_{3}$ is zero.

We now show that $M_{2}=M_{3}=0$. Suppose that $M_{2}=0$ and $M_{3} \neq 0$ (the other case being symmetric). Since the sum of every column of $M$ is 1 and also $M_{2}=0$, we have that the sum of every column of $M_{4}$ is 1 . Therefore, the sum of all the entries of $M_{4}$ is $|\Lambda|-x$, which in turn implies that the sum of every row of $M_{4}$ is exactly 1 . We conlude that $M_{3}=0$. Therefore,

$$
\begin{aligned}
\sum_{k=1}^{x} c^{(k)} & =\sum_{k=1}^{x} \sum_{j=1}^{|\Lambda|} j \cdot m_{j, k}=\sum_{k=1}^{x} \sum_{j=1}^{x} j \cdot m_{j, k} \\
& =\sum_{j=1}^{x} j \cdot \sum_{k=1}^{x} m_{j, k}=\sum_{j=1}^{x} j=\frac{x(x+1)}{2} .
\end{aligned}
$$




$$
M=\left[\begin{array}{ccc|cc}
0.6 & 0.1 & 0.1 & 0 & 0.2 \\
0 & 0.4 & 0.6 & 0 & 0 \\
0.1 & 0.5 & 0 & 0.3 & 0.1 \\
\hline 0.1 & 0 & 0.1 & 0.7 & 0.1 \\
0.2 & 0 & 0.2 & 0 & 0.6
\end{array}\right] \quad M^{\prime}=\left[\begin{array}{ccc|cc}
0.6 & 0.1 & 0.1 & 0 & 0.2 \\
0 & 0.4 & 0.6 & 0 & 0 \\
0.3 & 0.5 & 0 & 0.1 & 0.1 \\
\hline 0.1 & 0 & 0.1 & 0.7 & 0.1 \\
0 & 0 & 0.2 & 0.2 & 0.6
\end{array}\right]
$$

Figure 3: Consider the matrix $M$ above and the case where $x=3$. Since $M_{2}$ and $M_{3}$ are not zero, we can pick two non-zero cells from $M_{2}$ and $M_{3}$ respectively, say $m_{3,4}$ and $m_{5,1}$. In this case, $\varepsilon=0.2$. We then decrease $m_{3,4}$ and $m_{5,1}$ by 0.2 , and increase $m_{3,1}$ and $m_{5,4}$ by 0.2 . The modified matrix $M^{\prime}$ is shown above. We can see that $\sum_{k=1}^{x} c^{(k)}=7.4>7=\sum_{k=1}^{x} c^{\prime(k)}$.

Since $m$ minimizes $\sum_{k=1}^{x} c^{(k)}$ the lemma follows.

We show that the analysis of Theorem 14 is tight by showing that the integrality gap of the linear program is not smaller than 2 .

Theorem 15. The integrality gap of the linear program used in $\mathcal{B}$ is at least 2 .

Proof. We construct an instance of the problem and determine the gap between the relaxed and integer versions of the linear program. Consider $n$ identical intervals, each belonging to one class. A solution of the relaxed program is $m_{j, i}=1 / n$ for all $j$ and $i$. Thus each unit segment gets the following skyline.

$$
\sum_{j=1}^{n} j \cdot m_{j, i}=\sum_{j=1}^{n} j \cdot \frac{1}{n}=\frac{n(n+1)}{2} \cdot \frac{1}{n}=\frac{n+1}{2} .
$$

However the optimal solution for the integer linear program has skyline $n$ for each unit segment leading to the following integrality gap.

$$
\frac{n}{(n+1) / 2}=\frac{2 n}{n+1}=2-\frac{2}{n+1}
$$

By increasing $n$, the gap can be made arbitrarily close to 2 . Therefore, the worst-case integrality gap cannot be strictly smaller than 2 . 


\section{Conclusion}

We initiated the study of online algorithms for the coloring problem SKYLINE. An immediate research direction is to extend the online algorithms for two cases: (i) each color can have an arbitrary capacity; and (ii) the cost of a color class is given by an arbitrary increasing function, i.e., for arbitrary $\lambda$. Another direction is to find a better competitive ratio for bounded length intervals. The other directions include determining if there is PTAS for the problem or whether the problem is APX-hard.

For the variant Permutation, it is desirable to obtain a stronger complexity result by determining whether the problem of permuting the color classes stays NP-hard if the given color classes correspond to an overall optimal solution. It would be interesting to determine whether approximation ratio better than 2 can be achieved for Permutation. One could also study online algorithms and other objective functions.

\section{References}

[1] U. Adamy and T. Erlebach. Online coloring of intervals with bandwidth. In WAOA, pages 1-12, 2003. doi:10.1007/978-3-540-24592-6_1.

[2] M. Alicherry and R. Bhatia. Line system design and a generalized coloring problem. In ESA, pages 19-30, 2003. doi:10.1007/ 978-3-540-39658-1_5.

[3] Y. Azar, A. Fiat, M. Levy, and N. S. Narayanaswamy. An improved algorithm for online coloring of intervals with bandwidth. Theor. Comput. Sci., 363(1):18-27, 2006. doi:10.1016/j.tcs.2006.06.014.

[4] A. Borodin and R. El-Yaniv. Online Computation and Competitive Analysis. Cambridge University Press, 1998.

[5] J. Chang, H. N. Gabow, and S. Khuller. A model for minimizing active processor time. Algorithmica, 70(3):368-405, 2014. doi:10.1007/ s00453-013-9807-y.

[6] J. Chang, S. Khuller, and K. Mukherjee. LP rounding and combinatorial algorithms for minimizing active and busy time. In SPAA, pages 118-127, 2014. doi:10.1145/2612669.2612689. 
[7] M. Chrobak and M. Slusarek. On some packing problem related to dynamic storage allocation. ITA, 22(4):487-499, 1988.

[8] M. Flammini, G. Monaco, L. Moscardelli, H. Shachnai, M. Shalom, T. Tamir, and S. Zaks. Minimizing total busy time in parallel scheduling with application to optical networks. Theor. Comput. Sci., 411(4042):3553-3562, 2010. doi:10.1016/j.tcs.2010.05.011.

[9] M. Garey and D. S. Johnson. Computers and Intractability: A Guide to the Theory of NP-Completeness. W. H. Freeman, 1979.

[10] M. Garey, D. S. Johnson, G. Miller, and C. H. Papadimitriou. The complexity of coloring circular arcs and chords. SIAM J. Algebraic Discrete Methods, 1(2):216-227, 1980. doi:10.1137/0601025.

[11] M. M. Halldórsson, G. Kortsarz, and H. Shachnai. Minimizing average completion of dedicated tasks and interval graphs. In APPROX, pages 114-126, 2001. doi:10.1007/3-540-44666-4_15.

[12] K. Jansen. Approximation results for the optimum cost chromatic partition problem. J. Algorithms, 34(1):54-89, 2000. doi:10.1006/jagm. 1999.1022.

[13] R. Khandekar, B. Schieber, H. Shachnai, and T. Tamir. Minimizing busy time in multiple machine real-time scheduling. In FSTTCS, pages 169-180, 2010. doi:10.4230/LIPIcs.FSTTCS. 2010.169.

[14] H. Kierstead and W. Trotter. An extremal problem in recursive combinatorics. Congressus Numerantium, 33:143-153, 1981.

[15] L. G. Kroon, A. Sen, H. Deng, and A. Roy. The optimal cost chromatic partition problem for trees and interval graphs. In $W G$, pages 279-292, 1996. doi:10.1007/3-540-62559-3_23.

[16] M. Kubale, editor. Graph Colorings. American Mathematical Society, 2004.

[17] V. Kumar and A. Rudra. Approximation algorithms for wavelength assignment. In FSTTCS, pages 152-163, 2005. doi:10.1007/11590156_ 12 . 
[18] G. Mertzios, M. Shalom, A. Voloshin, P. Wong, and S. Zaks. Optimizing busy time on parallel machines. Theor. Comput. Sci., 562:524-541, 2015. doi:10.1016/j.tcs.2014.10.033.

[19] S. Nicoloso, M. Sarrafzadeh, and X. Song. On the sum coloring problem on interval graphs. Algorithmica, 23(2):109-126, 1999. doi:10.1007/ PL00009252.

[20] S. V. Pemmaraju, R. Raman, and K. R. Varadarajan. Buffer minimization using max-coloring. In SODA, pages 562-571, 2004.

[21] R. Ren and X. Tang. Clairvoyant dynamic bin packing for job scheduling with minimum server usage time. In $S P A A$, pages 227-237, 2016. doi: 10.1145/2935764.2935775.

[22] M. Shalom, A. Voloshin, P. Wong, F. Yung, and S. Zaks. Online optimization of busy time on parallel machines. Theor. Comput. Sci., 560:190-206, 2014. doi:10.1016/j.tcs.2014.07.017.

[23] P. Winkler and L. Zhang. Wavelength assignment and generalized interval graph coloring. In SODA, pages 830-831, 2003. 